\title{
CUANTIFICACIÓN DE METANO ENTÉRICO SEGÚN ESTADO FISIOLÓGICO EN VACAS LECHERAS DE ALTA PRODUCCIÓN EN COSTA RICA
}

\author{
Johnny Montenegro-Ballestero ${ }^{1 / *}$, Eduardo Barrantes-Guevara ${ }^{2}$, Sylvia Ivankovich-Cruz ${ }^{3}$ \\ Palabras clave: Metano entérico; ganado de leche; pastoreo; trópico; SF6; gases con efecto invernadero. \\ Keywords: Enteric methane; dairy cows; grazing; tropics; SF6; greenhouse gases.
}

\begin{abstract}
RESUMEN
Esta investigación cuantifica, mediante la técnica del SF6, el metano entérico en vacas altamente productoras en una finca comercial. El objetivo fue determinar la emisión de metano entérico en vacas Holstein en diferentes estados fisiológicos en un sistema de producción de leche intensivo bajo pastoreo. Para ello, se seleccionaron 9 vacas Holstein que representaron la conformación típica del hato en edad $(5,7 \pm 0,6$ años), número de lactancias $(3,6 \pm 0,4$ partos), producción promedio de leche por lactancia $(7924 \pm 280 \mathrm{~kg}$ leche) y peso $(672 \pm 36 \mathrm{~kg})$ en diferentes estados fisiológicos: secas (VS), preparto (VPP), primer tercio de lactancia (VPTL) $\mathrm{y}$ en medio/final ( $\mathrm{VM} / \mathrm{F})$ estado de lactación. Los forrajes y suplementeos proporcionados, previo al ordeño, fueron pesados y muestreados para determinar consumo y digestibilidad de la materia seca. En cada período de medición de metano, se realizaron muestreos de las pasturas y se determinó proteína cruda $(15,9 \pm 0,3 \%)$, fibra neutro detergente $(63,0 \pm 0,9 \%)$ y digestibilidad in vitro de la materia seca $(67,8 \pm 1,9 \%)$ del forraje

\footnotetext{
* Autor para correspondencia. Correo electrónico: jmontenegro@inta.go.cr

1 Instituto Nacional de Innovación y Transferencia en Tecnología Agropecuaria (INTA)-Instituto Meteorológico Nacional (IMN), Costa Rica.
} (D) 0000-0001-8526-570X.
\end{abstract}

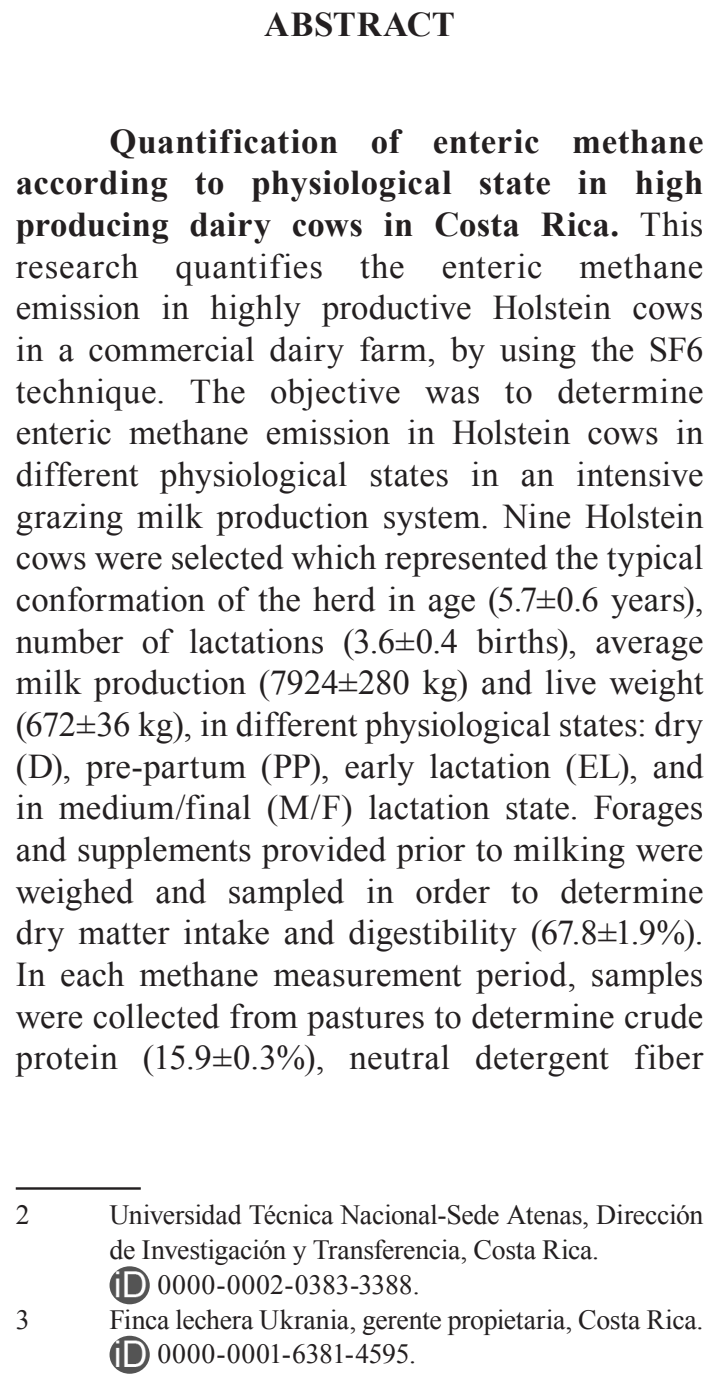


en oferta. La emisión diaria de metano por vaca fue similar $(p>0,05)$ en VS, VPP y en VM/F, y mayores $(p<0,05)$ en VPTL, con rangos $\left(g . \mathrm{V}^{-1} \cdot \mathrm{d}^{-1}\right)$ entre 240 y 443 en VS, de 238 a 483 en VPP, de 277 a 506 en VPTL, y de 281 a 470 en VM/F. Se determinó la relación lineal entre el consumo total de materia seca y la emisión de metano $\left(\mathrm{r}^{2}\right.$ varió entre 0,42 y 0,78 según el estado fisiológico), así como también entre el metano producido y la producción de leche por día $\left(\mathrm{r}^{2}=0,71\right)$. Las vacas más productoras emiten más metano, pero son menos emisoras de metano por litro de leche producida. El $\mathrm{P}_{\mathrm{m}}$ varió conforme al estado fisiológico y el valor anual fue similar al sugerido por el IPCC.

\section{INTRODUCCIÓN}

El cambio climático que se produce por el incremento de la concentración de gases con efecto invernadero (GEI) en la atmósfera, es una de las principales amenazas a las que se enfrenta hoy el planeta. De acuerdo con el Panel Intergubernamental de Cambio Climático (IPCC 2006), la temperatura media de la Tierra podría incrementarse entre 2 y $6^{\circ} \mathrm{C}$ con lo cual se comprometería la sobrevivencia de diferentes especies, vegetales y animales, incluido el ser humano.

Uno de los gases con efecto invernadero es el metano $\left(\mathrm{CH}_{4}\right)$, que en la ganadería se produce como resultado de la digestión anaeróbica de la materia orgánica. Este gas posee un potencial de calentamiento 21 veces mayor al $\mathrm{CO}_{2}$ y representa hasta el $18 \%$ de las emisiones mundiales de estos gases (Steinfeld et al. 2006). De hecho, su concentración atmosférica se ha incrementado considerablemente en el último siglo, al ser solo superado por el dióxido de carbono $\left(\mathrm{CO}_{2}\right)$.
$(63.0 \pm 0.9 \%)$ and in vitro dry matter digestibility. Daily methane emission per cow was similar $(\mathrm{p}>0.05)$ in $\mathrm{D}$, PP and in $\mathrm{M} / \mathrm{F}$, and higher $(\mathrm{p}<0.05)$ in EL, with ranges $\left(\mathrm{g} \cdot \mathrm{c}^{-1} \cdot \mathrm{d}^{-1}\right)$ between 240 and 443 in D, from 238 to 483 in PP, from 277 to 506 in EL, and from 281 to 470 in M/F. A linear relationship was determined between total dry matter intake and methane emission $\left(\mathrm{r}^{2}\right.$ varied between 0.42 and 0.78 depending on physiological state), as well as between methane produced and milk production per day $\left(\mathrm{r}^{2}=0.71\right)$. High producing cows emit more methane but are less methane emitters per liter of milk produced. The $\mathrm{Y}_{\mathrm{m}}$ varied depending on physiological state of the cows, and the annual value was similar to that suggested by IPCC.

El $\mathrm{CH}_{4}$ es uno de los productos finales de la fermentación microbiana por la acción de las arqueobacterias que utilizan el $\mathrm{H}_{2}$ para reducir el $\mathrm{CO}_{2}$, formándose en este proceso $\mathrm{CH}_{4} \mathrm{y} \mathrm{H}_{2} \mathrm{O}$, lo cual puede representar la pérdida de hasta el $14 \%$ de la energía digestible consumida (Cottle et al. 2011). Por ello, la reducción de esta pérdida de energía tendría consecuencias positivas tanto para el productor como para el ambiente.

La formación de metano en el rumen tiene relación directa con la calidad de la dieta consumida y, en sistemas de pastoreo, la calidad del pasto tiene influencia directa en la emisión de este gas. En este sentido, si las pasturas son de baja calidad nutritiva, se aumenta la metanogénesis ruminal, porque proporcionan niveles altos de fibra que reducen la tasa de paso, incrementado la fermentación y la producción de ácido acético e hidrógeno (Hammond et al. 2011). Por esta razón, los forrajes jóvenes de mejor calidad nutritiva que los maduros, y los alimentos balanceados modifican la fermentación ruminal, al producir proporcionalmente más 
ácido propiónico (Moss et al. 2000), disminuyen el pH ruminal, así como, reducen la población de los protozoos y la actividad fibrolítica (Martin et al. 2010) y con ello la producción y emisión de metano entérico. Por ello, el consumo, tipo y composición del alimento afectan la fermentación en el rumen y la emisión de este gas.

Con respecto al consumo de materia seca, diferentes investigaciones han señalado que a mayor consumo, mayor emisión de metano (Buddle et al. 2011, Moorby et al. 2015). Sin embargo, la cantidad de este gas que se produce, por unidad de consumo de materia seca, disminuye (Hammond et al. 2011, Chaokaur et al. 2015). Por su parte, la composición del alimento consumido influye en la emisión de $\mathrm{CH}_{4}$, debido a la relación con la tasa de pasaje, la cual se incrementa con la calidad nutritiva y ello reduce la generación de este gas en el sistema digestivo por unidad de alimento consumido (Knapp et al. 2014). Por ello, las dietas que contienen alta proporción de granos disminuyen la producción de metano en comparación con las dietas basadas en forraje (Beauchemin y McGinn 2005, Hammond et al. 2011).

Ya que en Costa Rica los sistemas especializados en la producción de leche que se ubican a más de $1000 \mathrm{msnm}$, incluyen la suplementación con granos como parte normal del manejo alimenticio del hato, es importante determinar la emisión de metano entérico en estos sistemas productivos.

Hasta la fecha, en Costa Rica existen muy pocas experiencias previas reportadas para la determinación directa de metano. Una de ellas es bajo condiciones de estabulación (Montenegro et al. 2016), otra en pastoreo (Montenegro et al. 2018) con bovinos de carne. De igual forma, Abarca et al. (2018) reportaron las emisiones para lechería tropical de bajura en condiciones de pastoreo, pero en una zona ecológica y con un material genético diferente al utilizado en esta investigación; por lo que esta representa la primera evaluación de campo para cuantificar el $\mathrm{CH}_{4}$ emitido por el ganado lechero especializado de altura. Datos anteriores a los referenciados para Costa Rica provienen de estimaciones que utilizaron las metodologías del IPCC.

De acuerdo con el Inventario Nacional de GEI de Costa Rica para el 2012, del total del metano emitido por el sector agropecuario, alrededor de $22 \%$ es responsabilidad del sector lechero por la fermentación entérica. Sin embargo, esta información ha sido desarrollada basada en la utilización de ecuaciones matemáticas y no en mediciones de campo.

Los datos generados en esta investigación proporcionan los primeros valores reales obtenidos por medición directa y relacionada con una condición específica de manejo bovino de lechería especializado de altura en pastoreo en Costa Rica. Esto es necesario para poder reportar datos, a la Comisión Marco de Cambio Climático de las Naciones Unidas, que sean representativos para el país.

Debido a que Costa Rica se ha planteado el objetivo de ser Carbono-Neutro para el 2021, la cuantificación de la emisión de este gas en los sistemas de producción de leche contribuirá de manera significativa a conocer la realidad de la emisión GEI de este sector y la potencial necesidad de desarrollar opciones de mitigación para esta actividad.

Basado en lo anterior, se realizó la presente investigación con el objetivo de cuantificar la emisión de metano entérico en vacas Holstein, altamente productoras en pastoreo y en diferentes estados fisiológicos, en un sistema de producción de leche intensivo de altura.

\section{MATERIALES Y MÉTODOS}

Esta investigación se desarrolló en una finca comercial lechera convencional especializada de altura (1700 msnm) con pastoreo intensivo ubicada en Cot de Cartago, Costa Rica, la cual fue seleccionada por su representatividad. La temperatura y precipitación anual promedio es de $16^{\circ} \mathrm{C}$ y $2700 \mathrm{~mm}$, respectivamente. Existe una época seca bien marcada con duración promedio de 4 meses y medio, que se extiende desde 
mediados de enero hasta finales de mayo, y la zona según la clasificación de Holdridge (1989) pertenece a bosque húmedo montano bajo. Los suelos son de origen volcánico, franco arcillosos con topografía ondulada.

Las pasturas en esta finca están compuestas por la mezcla de estrella africana (Cynodon nlemfuensis) y kikuyo (Kikuyuochloa clandestina) en proporción 50:48, respectivamente (la diferencia son malezas), manejadas con ciclos de pastoreo de medio día y 22 días de descanso, y con apartos de 3500 metros cuadrados a los cuales se les aplica fertilizante nitrogenado en dosis de $450 \mathrm{~kg} \cdot \mathrm{ha}^{-1}$.año ${ }^{-1}$ (urea, 46\% N) luego de la salida de las vacas en pastoreo (carga animal de 7,1 U.A. ha ${ }^{-1}$ ).

El hato es de la raza Holstein y las vacas son ordeñadas 2 veces al día, las cuales reciben suplementación según el estado fisiológico, y el respectivo al nivel productivo. Para esta investigación, se seleccionaron 9 vacas que representaron la conformación promedio del hato de la finca, es decir, se consideró la edad (5,7 $\pm 0,6$ años), el número de lactancias $(3,6 \pm 0,4$ partos), producción de leche por lactancia $(7924 \pm 280 \mathrm{~kg})$ y peso $(672 \pm 36 \mathrm{~kg})$; de manera que, se obtuvo un grupo proporcionalmente similar al hato total manejado en la finca. Esta condición es muy importante, debido a que los resultados serán extrapolables al sistema de producción de leche especializado de altura, por cuanto las fincas en general poseen una estructura de hato similar al evaluado.
El proceso de investigación se inició con vacas que recién finalizaban la producción de leche, a los 300 días después del parto en el estado fisiológico denominado seco. Este criterio fue de importancia, porque permitió entrenar a las vacas al manejo experimental, en un momento en el cual no se afectaba la producción de leche.

A este grupo de vacas se les dio seguimiento, en todas las determinaciones de metano que se realizaron en la finca; evaluaron las mismas vacas, lo cual fue de gran utilidad, ya que estaban entrenadas y acostumbradas al equipo utilizado para recolectar las muestras de gas sin causar modificación alguna de comportamiento y de manejo. Este grupo de animales pastoreó con el resto de vacas en la finca, según el estado fisiológico en el que se encontraba cada una al momento de realizar las evaluaciones.

En el manejo habitual de esta finca productora, y como norma en las fincas especializadas de producción de leche, el nivel de suplementación se ajusta de acuerdo con el estado fisiológico y producción de leche (Tabla 1). En la finca seleccionada, la producción de leche es muy estable en la fase media y final de la lactación, por lo que el cambio en la cantidad del alimento balanceado suministrado a las vacas es mínimo. Las vacas secas y en pre-parto reciben la suplementación una vez al día en el trascurso de la mañana, mientras que para las vacas en producción se divide la suplementación en 2 partes iguales, las que se proporcionan previo a cada ordeño.

Tabla 1. Suplementación diaria, kg MS, ofrecida a cada vaca según estado fisiológico.

\begin{tabular}{|c|c|c|c|c|}
\hline Componente & Secas $^{1}$ & Pre-parto ${ }^{1}$ & $\begin{array}{l}\text { Primer tercio } \\
\text { de lactancia }{ }^{1}\end{array}$ & Media/final $^{1}$ \\
\hline Alimento balanceado & $1,7 \pm 0,1$ & $2,2 \pm 0,1$ & $7,9 \pm 0,1$ & $6,8 \pm 0,2$ \\
\hline Maíz molido & --- & $1,7 \pm 0,1$ & $0,6 \pm 0,1$ & $1,1 \pm 0,1$ \\
\hline Pelets ${ }^{2}$ & --- & $0,8 \pm 0,1$ & $1,6 \pm 0,1$ & $1,8 \pm 0,1$ \\
\hline Harina de soja & --- & $0,3 \pm 0,1$ & $1,4 \pm 0,1$ & --- \\
\hline Silopaca & $1,3 \pm 0,1$ & $0,4 \pm 0,2$ & $2,4 \pm 0,3$ & $3,0 \pm 0,2$ \\
\hline Paja de arroz & $0,7 \pm 0,1$ & --- & $0,2 \pm 0,1$ & --- \\
\hline
\end{tabular}

1 Estado fisiológico; Valores promedio \pm 1 error estándar (EE).

2 Residuo de planta de jugo de naranja. 
Las vacas secas y en pre-parto se manejaron juntas, pero separadas del hato en producción y fueron llevadas diariamente a un galpón donde recibieron la suplementación (Tabla 1). A estas vacas se les proporcionó el manejo normal de la finca y se mantuvieron con el resto de las vacas en estos estados fisiológicos.

Las vacas en el primer tercio de lactancia y en media/final lactación, que se utilizaron para la cuantificación de la emisión de $\mathrm{CH}_{4}$ entérico, siguieron la rutina de manejo establecida en la finca, de manera que pastorearon con el resto del hato en producción y recibieron la dieta que normalmente se les proporciona de acuerdo con el estado fisiológico de las mismas (Tabla 1).
Los ordeños diarios se realizaron a las 2 am y $2 \mathrm{pm}$, momento en el cual se les proporcionó el suplemento (Tabla 1), el cual fue pesado de manera individual para cada una de las vacas utilizadas para la determinación de la emisión de metano entérico durante el período de recolección de las muestras de metano. Los suplementos y forrajes proporcionados en la canoa, previo al ordeño, fueron muestreados para determinar la digestibilidad in vitro de la materia seca (DIVMS, Tabla 2). Los contenidos de proteína $(\mathrm{PC}, \%)$ y fibra neutro detergente (FND, \%) de los granos fueron tomados directamente de las etiquetas de los productos comerciales, mientras que a los forrajes se les determinó estos contenidos vía análisis de laboratorio.

Tabla 2. Calidad nutritiva de la suplementación suministrada a las vacas.

\begin{tabular}{lcccc}
\hline \multicolumn{1}{c}{ Componente } & $\begin{array}{c}\text { PC, } \\
\%\end{array}$ & $\begin{array}{c}\text { FND, } \\
\%\end{array}$ & $\begin{array}{c}\text { ED, } \\
\mathrm{kcal.kg}^{-1} \mathrm{MS}\end{array}$ & $\begin{array}{c}\text { DIVMS, } \\
\%\end{array}$ \\
\hline${\text { Alimento }{ }^{*}}^{*}$ & 13,0 & 10,0 & 3200 & $82,2 \pm 1,0$ \\
balanceado $^{2 *}$ & 16,0 & 10,0 & 3400 & $78,9 \pm 0,6$ \\
Maíz molido* $^{*}$ Pelets $^{*}$ & 7,0 & 4,0 & 3500 & $80,7 \pm 1,4$ \\
Harina de soja $^{*}$ & 12,0 & 22,0 & 3000 & $71,8 \pm 1,4$ \\
Silopaca & 46,5 & 4,5 & 3300 & $50,0 \pm 2,9$ \\
Paja de arroz & $5,2 \pm 0,6$ & $22,2 \pm 0,8$ & & $36,8 \pm 1,3$ \\
\hline
\end{tabular}

Valores promedio \pm EE corresponden a análisis realizados en INTA.

1 Para vacas secas y en preparto.

2 Para vacas en producción.

* Valores tomados de la etiqueta del producto.

En cada período de medición de metano, se estimó de manera lineal (Elizondo 2002) el consumo total de materia seca para cada vaca, al cual se le sustrajo lo aportado por el suplemento diario ofrecido (Tabla 1). La diferencia se asumió como parte de la actividad realizada durante el pastoreo.

En cada período de determinación de metano, en 3 apartos se realizaron muestreos de pastoreo simulado al recolectar 5 muestras en cada uno de ellos, para precisar proteína cruda (PC), fibra neutro detergente (FND) y digestibilidad (DIVMS) del forraje en oferta. Los análisis se realizaron por duplicado en el laboratorio de Forrajes del Instituto Nacional de Innovación y Transferencia en Tecnología Agropecuaria (INTA). Para cada variable los valores fueron similares y los resultados promedio fueron: PC: $15,9 \pm 0,3 \%$, FND: $63,0 \pm 0,9 \%$, y DIVMS: $67,8 \pm 1,9 \%$.

La cuantificación de la emisión del metano entérico, se realizó mediante la selección de 
diferentes estados fisiológicos, los mismos que se identifican en el ciclo anual productivo de la vaca lechera y se describen a continuación:

I. Vacas secas (VS) $=15$ días después de dejar de ordeñarse.

II. Vacas pre-parto (VPP) $=45$ después de dejar de ordeñarse.

III. Vacas primer tercio de lactancia (VPTL) $=$ $43 \pm 8$ días de paridas.

IV. Vacas en media/final lactación $(\mathrm{VM} / \mathrm{F})=$ $180 \pm 8$ días de paridas.

Para determinar la emisión de $\mathrm{CH}_{4}$, se utilizó la técnica de hexafluoruro de azufre, la cual ha sido previamente implementada por Montenegro y Barrantes (2016) en Costa Rica. Las mediciones se realizaron entre marzo y noviembre.

Durante el desarrollo de la evaluación, previo a cada período de determinación de la emisión del $\mathrm{CH}_{4}$, a las vacas se les dio una fase de adaptación de 5 días para que se acostumbraron a llevar el equipo recolector del gas. Cada período de cuantificación de la emisión de $\mathrm{CH}_{4}$ consistió en 5 días consecutivos ( 24 h y de lunes a sábado) en los que se intercambiaron los yokes a la misma hora cada día.

En cada período de muestreo, se recolectaron diariamente muestras de aire del aparto para conocer los niveles normales de $\mathrm{CH}_{4}$ y SF6 en el ambiente. Estos valores fueron sustraídos de los resultados obtenidos con las muestras de gas recolectados en los yokes.

Las muestras gaseosas fueron analizadas en el Laboratorio de Suelos del INTA, utilizando un cromatógrafo de gases (modelo Agilent 7890A; Santa Clara, CA, Estados Unidos) equipado con 2 detectores: ionización de llama y de captura de electrones.

La concentración de $\mathrm{CH}_{4}$ y de SF6, en las muestras gaseosas se determinó por interpolación a partir de curvas de calibración construidos con estándares de concentración conocida y trazable a NIST (Scott-Marrin, Inc., Ca,); como control de calidad se analizaron intercaladamente muestras estándar con las provenientes del campo.

Con la tasa de liberación conocida del SF6, proveniente de las cápsulas y la concentración de $\mathrm{CH}_{4}$ y SF6 de las muestras analizadas, se calculó la emisión de $\mathrm{CH}_{4}$ para cada vaca como el producto de la tasa de emisión y el cociente de $\mathrm{CH}_{4}$ y SF6 en la muestra. Para obtener la emisión entre períodos donde no se realizaron mediciones de metano, se realizó inferencia lineal entre las determinaciones realizadas a cada una de las vacas del experimento. De esta manera, la emisión diaria obtenida se multiplicó por el número de días del mes respectivo y se totalizó para todo el año.

Finalmente, se comparó la emisión de metano de los estados fisiológicos por medio de la diferencia mínima significativa en InfoStat (Di Rienso et al. 2015) y se realizaron análisis de correlación entre consumo de materia seca y emisión por vaca por día, así también, entre la producción de leche por vaca por día y la emisión de metano por kilogramo de leche, y entre el consumo de materia seca y la producción de metano $\left(\mathrm{P}_{\mathrm{m}}\right)$ en porcentaje, que es otra forma de expresar la emisión de metano en bovinos. El $\mathrm{P}_{\mathrm{m}}$ es la relación entre la cantidad de metano emitido y el consumo de alimento, ambos expresados en términos de energía de combustión (Lassey 2007). Por ejemplo, el IPCC (2006) sugiere un valor típico de $\mathrm{P}_{\mathrm{m}}$ de $6,5 \%$ para $\mathrm{CH}_{4}$ entérico emitido por bovinos en países tropicales.

\section{RESULTADOS}

\section{Emisión de metano entérico según estado fisiológico}

La emisión de metano entérico por vaca mostró similar magnitud ( $p>0,05)$ en VS, VPP $y$ en $V M / F$, al ser mayores $(p<0,05)$ las que se presentaron en VPTL (Figura 1). 


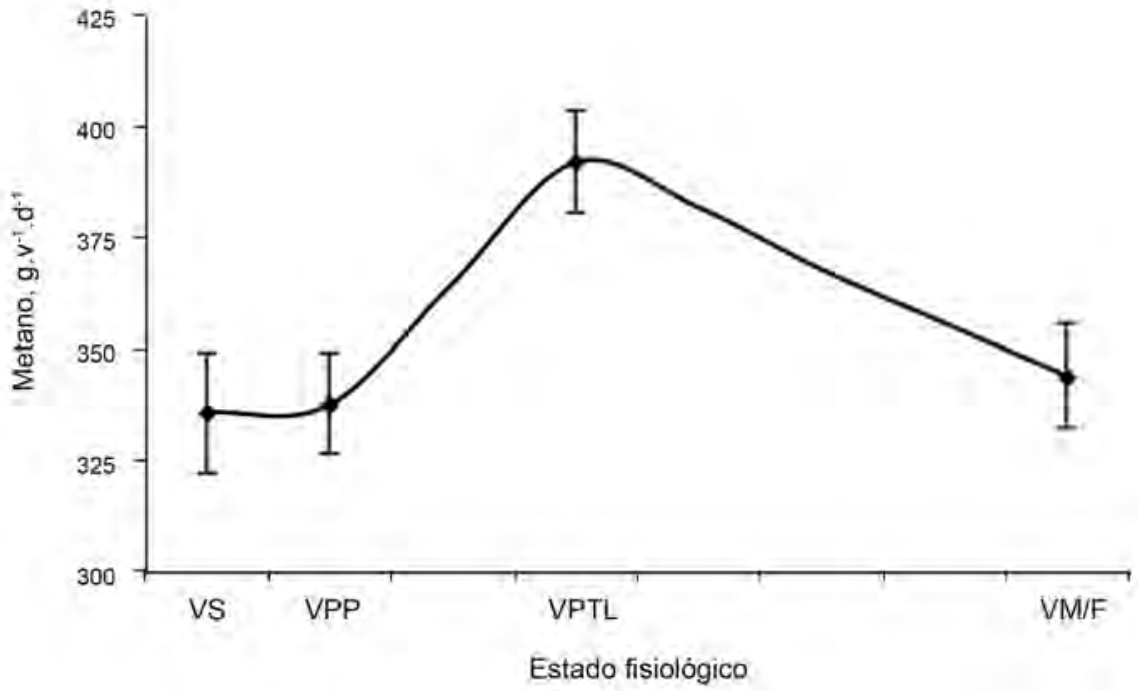

Valores de emisión $\pm 1 \mathrm{EE}$

$\mathrm{VS}=$ vacas secas, $\mathrm{VPP}=$ vacas pre-parto, $\mathrm{VPTL}=$ vacas en primer tercio de lactancia, $\mathrm{VM} / \mathrm{F}=$ vacas

Figura 1 Emisión de metano entérico, g.d $\mathrm{d}^{-1}$, en vacas lecheras Holstein según estado fisiológico.

La emisión determinada presentó rangos, los cuales son el resultado del peso vivo, el cual está relacionado con la edad de las vacas lecheras utilizadas en el experimento.

\section{Relación emisión de metano y consumo de materia seca}

El consumo voluntario es uno de los factores que influyen en la emisión de este gas, al existir relación directa entre el consumo total y la emisión de metano (Figura 2, Tabla 3). 

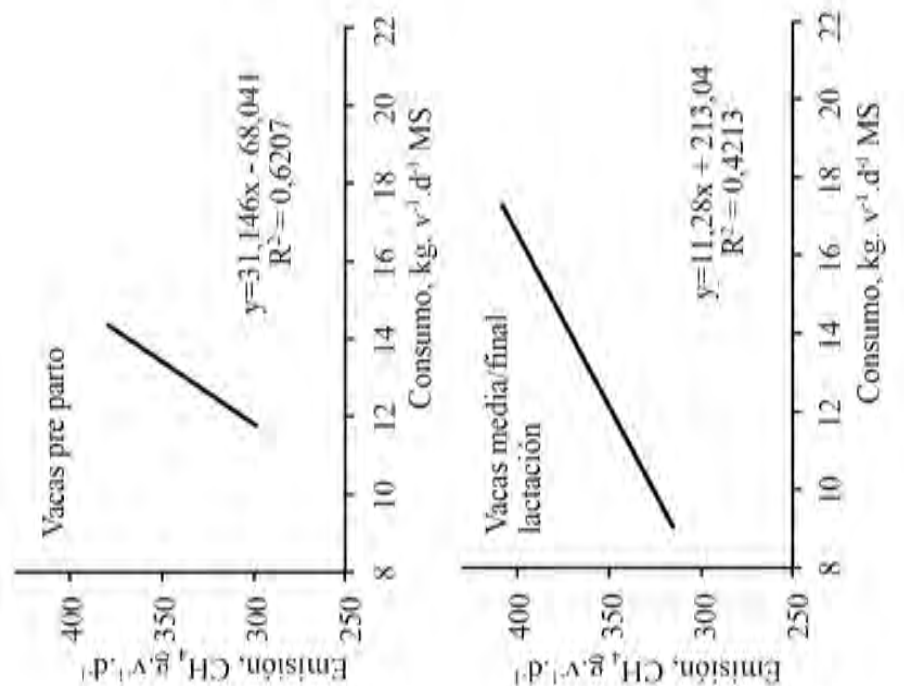
Tabla 3. Consumo de materia seca (MS), producción de leche y metano emitido en vacas Holstein en diferentes estados fisiológicos.

\begin{tabular}{lcccc}
\hline \multicolumn{1}{c}{ Variable } & $\mathrm{VS}^{*}$ & $\mathrm{VPP}^{*}$ & $\mathrm{VPTL}^{*}$ & $\mathrm{VM}^{*} \mathrm{~F}^{*}$ \\
\hline Consumo, $\mathrm{kg} \cdot \mathrm{v}^{-1} \cdot \mathrm{d}^{-1} \mathrm{MS}$ & $11,7 \pm 0,4$ & $12,1 \pm 0,7$ & $19,4 \pm 0,9$ & $15,6 \pm 1,1$ \\
Leche, $\mathrm{kg} \cdot \mathrm{v}^{-1} \cdot \mathrm{d}^{-1}$ & --- & --- & $35,9 \pm 3,4^{\mathrm{a}}$ & $26,3 \pm 3,2^{\mathrm{b}}$ \\
$\mathrm{CH}_{4}, \mathrm{~kg} \cdot \mathrm{v}^{-1} \cdot \mathrm{d}^{-1}$ & $0,33 \pm 0,1$ & $0,34 \pm 0,1$ & $0,39 \pm 0,1$ & $0,34 \pm 0,1$ \\
$\mathrm{CH}_{4}, \mathrm{~g} \cdot \mathrm{kg}^{-1} \mathrm{MS}$ & $27,5 \pm 1,5$ & $28,5 \pm 2,0$ & $20,5 \pm 1,3$ & $21,1 \pm 1,1$ \\
$\mathrm{CH}_{4}, \mathrm{~g} \cdot \mathrm{kg}^{-1}$ leche & --- & --- & $11,6 \pm 1,6^{\mathrm{b}}$ & $14,2 \pm 2,0^{\mathrm{a}}$ \\
\hline $\mathrm{P}_{\mathrm{m}}, \%$ & $8,1 \pm 0,4$ & $7,8 \pm 0,2$ & $6,2 \pm 0,4$ & $6,7 \pm 0,4$
\end{tabular}

${ }^{*} \mathrm{VS}$ = vacas secas; VPP: en pre parto; VPTL = primer tercio de lactancia; VM/F = media/final lactación.

Medias con diferente letra difieren al nivel $\mathrm{p}<0,05$.

En todos los casos se determinó correlación lineal entre estas variables, al ser menor la variación en las VS y VPP (Figura 2, Tabla 3). Las diferencias en el peso de las vacas, luego del parto, explican la mayor variabilidad observada en las vacas en producción.

En el caso de VS y VPP, al estar gestantes y con un ternero en desarrollo en su última fase de gestación, hace que este ocupe una parte importante de la cavidad abdominal, lo cual reduce las posibilidades de expansión del rumen, y en consecuencia, el consumo disminuye (Mejía 2002) y es poco variable. De hecho, estos 2 grupos de vacas presentaron similar consumo (Tabla 3 ), pero menor al determinado en las vacas en producción. Esto apoya el hecho de que estas últimas, por no estar con limitación de distención del rumen, presenten mayor consumo y, por lo tanto, hace que la emisión total diaria de metano se incremente como consecuencia de mayor cantidad de sustrato fermentado en el rumen.

El mayor consumo de materia seca observado en las vacas en producción (VPTL y $\mathrm{VM} / \mathrm{F}$ ), con respecto al período seco y preparto, es congruente con el comportamiento normal del consumo durante el ciclo productivo de las vacas lecheras. El mayor consumo de las vacas, en el primer tercio de lactancia, resultó en emisiones diarias de metano que fueron $14 \%$ más altas que las determinadas en las vacas secas y en preparto. De igual manera, resultaron ser $12 \%$ mayores que durante la fase media/final de la lactancia.

\section{Eficiencia de emisión}

La eficiencia de emisión, la cual se refiere a la cantidad de metano, emitida por $\mathrm{kg}$ de leche producida, fue mejor $(\mathrm{p}<0,05)$ en las vacas en el primer tercio de lactancia que en las que se encontraban en media/final estado de lactación (Tabla 3). La mayor producción de leche de las VPTL hace que esta relación sea más baja a pesar del hecho de producir más metano (Figura 1) que las VM/F lactación.

La totalidad de los datos obtenidos durante los períodos muestreados con las vacas en producción (Figura 3) muestran claramente la relación lineal $\left(\mathrm{r}^{2}=0,71\right)$ existente, donde las vacas más productoras son menos emisoras de metano por kilogramo de leche producida. 


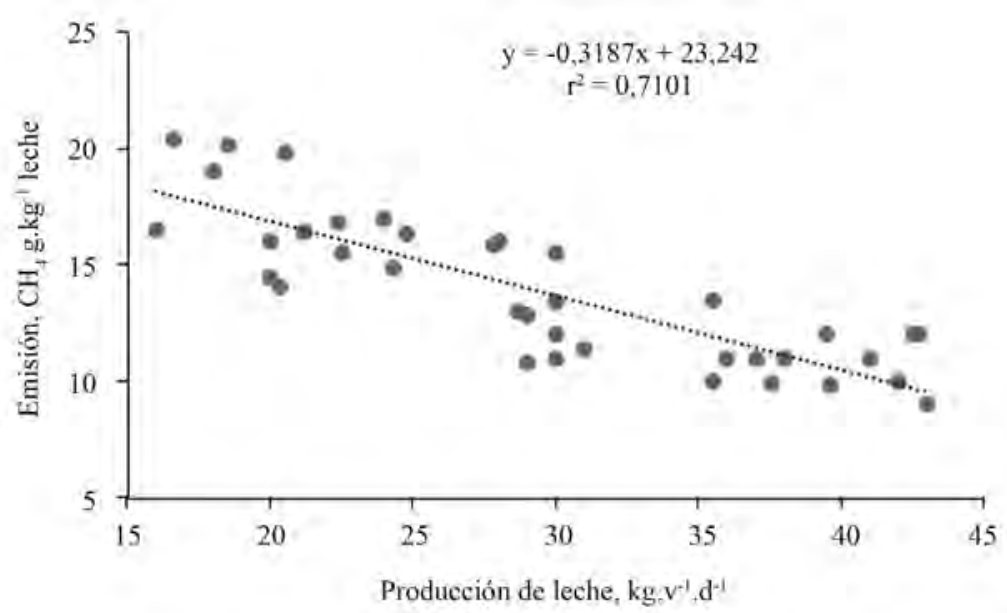

Figura 3 Relación entre emisión de metano entérico y la producción de leche en vacas lecheras Holstein.

La eficiencia de emisión determinada en esta investigación demuestra, claramente, que es posible desarrollar un enfoque de mitigación viable mediante la productividad, por lo que se satisface al mismo tiempo la creciente demanda de leche (Gerber et al. 2011) y los requerimientos ambientales ligados al cambio climático.

\section{Factor de conversión}

Una forma alternativa de expresar la emisión de metano entérico es mediante el factor de conversión para la producción de metano ( $\mathrm{Y}_{\mathrm{m}}$ por sus siglas en inglés o $\mathrm{P}_{\mathrm{m}}$ en español), que relaciona el metano emitido por unidad de consumo, ambas variables expresadas en términos de energía de combustión y se expresa como porcentaje de la energía bruta (GE) consumida (Lassey 2007).

De acuerdo con lo anterior, se determinó que el consumo está relacionado con el $\mathrm{P}_{\mathrm{m}}$, de manera que, aquellas vacas que tienen mayor consumo presentan menor porcentaje de la energía transformada y perdida como metano (Figura 4, Tabla 3), lo cual muestra que estas vacas son muy eficientes en la utilización de la energía contenida en la dieta, tal y como se demostró en la Figura 3. El $\mathrm{P}_{\mathrm{m}}$ determinado en esta investigación varió dependiendo del estado fisiológico de las vacas (Tabla 3 ) siendo más eficientes las vacas en producción, particularmente las VPTL, posiblemente influyendo en esto la cantidad de grano suministrado (Tabla 1). 


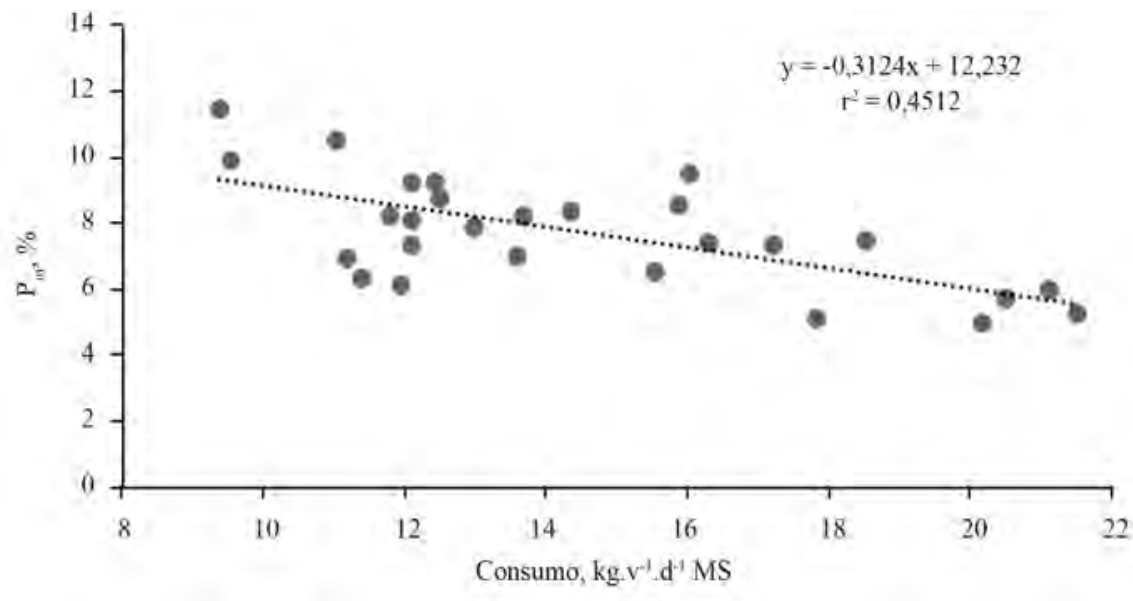

Figura 4 Relación entre consumo y $\mathrm{P}_{\mathrm{m}}$ en vacas lecheras Holstein.

\section{Emisión anual}

La emisión anual de metano estimada fue de $128,7 \mathrm{~kg} \cdot \mathrm{v}^{-1}$, la cual, de acuerdo con las guías metodológicas para la preparación de inventarios de gases del IPCC (2006), vacas con producciones anuales alrededor de los 8.000 kilos de leche presentarían una emisión de $121 \mathrm{~kg}$ de metano, por ello el $\mathrm{P}_{\mathrm{m}}$ anual determinado también es similar al sugerido por el IPCC. Para América Latina, el IPCC tiene como valor referenciado en vacas lecheras con producciones anuales de 800 kilogramos con una emisión de metano de $65 \mathrm{~kg}$. Es por esa razón es que se realiza la comparación con el dato antes referenciado del norte del continente. Esto demuestra que los datos que utiliza el IPCC como referencia no necesariamente representan las condiciones productivas de los sistemas y esto refuerza la necesidad de realizar este tipo de investigaciones.

Al comparar la producción de leche promedio $(7924 \pm 280 \mathrm{~kg})$ de las vacas evaluadas con el promedio productivo por vaca $(3500-4000 \mathrm{~kg}$ ) en sistemas de producción similares ubicados en esta región (R. Gutiérrez, 2015. Comunicación personal), se determina que se requieren 2,1 vacas para poder obtener la misma cantidad de leche. Debido a que no existe información de emisión de metano determinada bajo condiciones de finca para la región donde se realizó esta evaluación, la siguiente comparación se realizó con los resultados obtenidos al utilizar la metodología propuesta por el IPCC (2006) para el Nivel II.

Se estimó la emisión de metano en aproximadamente $84 \mathrm{~kg}$ por vaca por año, lo cual significa que en promedio se estarían emitiendo al ambiente un total adicional de $48,8 \mathrm{~kg}$ de metano por año, para producir la misma cantidad de leche de las vacas evaluadas. Por lo tanto, debido a la mayor eficiencia de las vacas altamente productoras en las cuales se determinó la emisión del metano entérico, por cada $\mathrm{kg}$ de leche están mitigando un total de $6,2 \mathrm{~g}$ de metano, lo que en términos porcentuales significa que emite $27,5 \%$ menos.

\section{DISCUSIÓN}

La eficiencia de emisión de metano entérico (g. $\mathrm{kg}^{-1}$ de leche), determinada en las vacas en el primer tercio de lactancia, presentó valores bajos y similares a los reportados en diferentes investigaciones realizadas en Chile, Uruguay, Canadá y el Reino Unido (Muñoz et al. 2015, 
Dini et al. 2012, Grainger et al. 2014, Jiao et al. 2014), quienes informan de un rango entre $10,8 \mathrm{y}$ 20,3 g. $\mathrm{kg}^{-1}$ de $\mathrm{CH}_{4}$ de leche para vacas Holstein en el pico de lactancia en Chile (Muñoz et al. 2015) o en media/final lactación en Uruguay (Dini et al. 2012). Las VS presentaron emisiones diarias que fueron similares a las reportadas por Garnsworthy et al. (2012) en el Reino Unido.

Esto demuestra que la eficiencia de utilización de la energía consumida por las vacas en la finca, donde se realizó la evaluación, es muy buena y que el consumo de granos contribuye a reducir la emisión de metano. Existen diferentes resultados de trabajos de investigación (Lovett et al. 2005, Jiao et al. 2014, Muñoz et al. 2015) que muestran que la suplementación con granos es una importante estrategia para reducir las emisiones de $\mathrm{CH}_{4}$ por unidad de consumo. Esto por supuesto, influye también en la eficiencia de emisión, tal y como se comentó, y muestra en las Tablas 1 y 3 .

En relación con la emisión diaria, esta presentó un rango entre 240 y 443 g. $\mathrm{v}^{-1}$ en VS, de 238 a 483 g. $v^{-1}$ en VPP, de 277 a 506 g. $v^{-1}$ en VPTL, y de 281 a 470 g.v - $^{-1}$ en VM/F, rangos que fueron similares a los reportados por Garnsworthy et al. (2012). Estos rangos diarios se explican tanto por diferencias en el peso vivo de las vacas evaluadas (entre 463 y $779 \mathrm{~kg}$ ), ya que ellas son una mezcla proporcional de edad (de 3 a 8 años) y representan típicamente la complejidad de fincas lecheras comerciales, como también por variaciones normales que ocurren día a día. Variaciones diarias en la emisión de metano son comúnmente informadas en los trabajos de investigación (Vlaming et al. 2005, Yan et al. 2010, Bell et al. 2014).

El peso vivo está directamente ligado al consumo y este a la emisión de metano, tal y como se demostró en esta investigación y también reportado en diferentes trabajos (Garnsworthy et al. 2012, Bell et al. 2014).

El incremento observado en la emisión de metano con el primer tercio de lactancia (VPTL) es congruente con el mayor consumo determinado en este grupo de vacas y la producción de leche obtenida, situación que ha sido previamente informada (Garnsworthy et al. 2012 y Bell et al. 2014). Durante esta etapa fisiológica, si bien se incrementa la emisión, debido a la gran producción de leche se reduce la emisión por unidad de producto, lo cual es reflejo del incremento de la eficiencia de utilización de la energía (Bell et al. 2014).

Con respecto al factor de emisión $\left(\mathrm{P}_{\mathrm{m}}\right)$, el promedio anual del $\mathrm{P}_{\mathrm{m}}$ fue de $6,7 \pm 0,5 \%$ el cual es similar al sugerido por el IPCC (2006) y a los reportados por Dini et al. (2012) para vacas Holstein $(6,6)$ en Uruguay. Muñoz et al. (2015) en Chile trabajando con vacas Holstein y proporcionar hasta $5 \mathrm{~kg}$ de alimento balanceado, reportaron un $\mathrm{P}_{\mathrm{m}}$ de 6,4, pero ligeramente superior al determinado por Jiao et al. (2014) en el Reino Unido para vacas lecheras Holstein $(5,4$ a 5,9).

\section{CONCLUSIONES}

Las vacas en el estado fisiológico seco, preparto y media/final etapa de lactación producen cantidades similares de metano entérico, al ser mayor la emisión en las vacas en el primer tercio de lactancia. Se determinó que conforme se incrementa el consumo de materia seca, también se aumenta la emisión diaria de metano, sin embargo, se reduce la emisión por unidad de materia seca consumida. Esto impacta de la misma forma el $\mathrm{P}_{\mathrm{m}}$, y la eficiencia de emisión (g. $\mathrm{kg}^{-1}$ de $\mathrm{CH}_{4}$ de leche), los cuales son mejores durante la lactancia temprana aspecto que demuestra que en ese estado fisiológico, las vacas son muy eficientes en la utilización del alimento consumido. Esta situación hace que vacas altamente productoras al recibir suplementación con alimentos balanceados, generen un producto amigable con el ambiente al lograr bajas emisiones por unidad de producto generado. Es por ello que se debe enfocar el manejo de las fincas hacia la productividad, debido a que se requiere menor número de animales para producir un determinado volumen de alimento, en este caso la leche, comparado con la obtención de la misma cantidad de leche, pero con vacas menos productoras 
(Gerber et al. 2011), además, propone un beneficio ambiental por la menor cantidad de metano producido y emitido a la atmósfera.

Es deseable, para próximas experiencias de esta naturaleza, que se establezca el consumo de materia seca mediante alguna técnica que lo permita determinar de manera directa, para cada uno de los animales en el experimento.

\section{AGRADECIMIENTOS}

Un agradecimiento especial a la señora Doris Ivankovich, dueña de la Finca Duraznal, por permitir el desarrollo de la presente investigación, lo cual fue clave poder generar la información que se presenta en este artículo.

Se agradece también al USAID, que a través del proyecto EC-LEDS II, financió parcialmente esta investigación, la cual fue realizada en el marco del Acuerdo interinstitucional entre el Instituto Nacional de Innovación y Transferencia en Tecnología Agropecuaria (INTA, Costa Rica) y el Instituto Meteorológico Nacional (IMN), en colaboración con la Universidad Técnica Nacional (UTN, Costa Rica), sede Atenas.

\section{LITERATURA CITADA}

Abarca, S; Soto, R; Villanueva, C. 2018. Emisión de metano por fermentación entérica en vacas bajo pastoreo de lechería tropical. Alcances Tecnológicos 12(2):45-53.

Beauchemin, K; McGinn, S. 2005. Methane emissions from feedlot cattle fed barley or corn diets. Journal of Animal Science 83:653-661.

Bell, M; Potterton, S; Craigon, J; Saunders, N; Wilcox, R; Hunter, M; Goodman, R; Garnsworthy, P. 2014. Variation in enteric methane emissions among cows on commercial dairy farms. Animal 8-9:1540-1546.

Buddle, B; Denis, M; Attwood, G; Altermann, E; Janssen, P; Ronimus, R; Pinares-Patiño, C; Muetzel, S; Wedlock, D. 2011. Strategies to reduce methane emissions from farmed ruminants grazing on pastures. The Vet. J. 188:11-17.

Chaokaur, A; Nishida, T; Phaowphaisal, I; Sommart, K. 2015. Effects of feeding level on methane emissions and energy utilization of Brahman cattle in the tropics. Agriculture, Ecosystems and Environment 199:225-230.
Charmley, E; Williams, S; Moate, P; Hegarty, R; Herd, R; Oddy, V; Reyenga, P; Staunton, K; Anderson, A; Hannah, M. 2016. A universal equation to predict methane production of forage-fed cattle in Australia. Animal Production Science 56:169-180.

Cottle, D; Nolan, J; Wiedemann, S. 2011. Ruminant enteric methane mitigation: a review. Animal Production Science 51:491-514.

Di Rienzo, J; Casanoves, F; Balzarini, M; Gonzalez, L; Tablada, M; Robledo, C. 2015. InfoStat versión 2015, Grupo InfoStat, FCA. Universidad Nacional de Córdoba, Argentina. URL http://www.infostat. com.ar

Dini, Y; Gere, J; Briano, C; Manetti, M; Juliarena, P; Picasso, V; Gratton, R; Astigarraga, L. 2012. Methane emission and milk production of dairy cows grazing pastures rich in legumes or rich in grasses in Uruguay. Animals 2:288-300.

Elizondo, J. 2002. Estimación lineal de los requerimientos nutricionales del NRC para ganado de leche. Agronomía Mesoamericana 13(1):41-44.

Garnsworthy, P; Craigon, J; Hernandez-Medrano, J; Saunders, N. 2012. Variation among individual dairy cows in methane measurements made on farm during milking. J. Dairy Sci. 95:3181-3189.

Gerber, P; Vellinga, T; Opio, C; Steinfeld, H. 2011. Productivity gains and greenhouse gas emissions intensity in dairy systems. Livestock Science 139:100-108.

Grainger, C; Clarke, T; McGinn, S; Auldist, M; Beauchemin, K; Hannah, M; Waghorn, G; Clark, H; Eckard, R. 2014. Methane emissions from dairy cows measured using the sulfur hexafluoride (SF6) tracer and chamber techniques. J. Dairy Sci. 90:2755-2766.

Hammond, K; Hoskin, S; Burke, J; Waghorn, G; Koolaard, J; Muetzel, S. 2011. Effects of feeding fresh white clover (Trifolium repens) or perennial ryegrass (Lolium perenne) on enteric methane emissions from sheep. Animal Feed Science and Technology 166-167:398-404.

Holdridge, L. 1989. Ecología basada en zonas de vida, Instituto Interamericano de Ciencias Agrícolas, San José, Costa Rica. 96 p.

IPCC (Intergovermental Panel on Climate change). 2006. Guidelines for National Greenhouse Gas Inventories. Prepared by the National Greenhouse Gas Inventories Programme, Vol, 4-1, IGES, Japan. $49 \mathrm{p}$.

Jiao, H; Dale, A; Carson, A; Murray, S; Gordon, A; Ferris, C. 2014. Effect of concentrate feed level on methane emissions from grazing dairy cows. J. Dairy Sci. 97:7043-7053

Knapp, J; Laur, G; Vadas, P; Weiss, W; Tricarico, J. 2014. Enteric methane in dairy cattle production: Quantifying the opportunities and impact of reducing emissions. J. Dairy Sci. 97:3231-3261. 
Lassey, K. 2007. Livestock methane emission: From the individual grazing animal through national inventories to the global methane cycle. Agricultural and Forest Meteorology 142:120-132.

Lovett, D; Stack, L; Lovell, S; Callan, J; Flynn, B; Hawkins, M; O’Mara, F. 2005. Manipulating enteric methane emissions and animal performance of late-lactation dairy cows through concentrate supplementation at pasture. Journal of Dairy Science 88:2836-2842.

Martin, C; Morgavi, D; Doreau, M. 2010. Methane mitigation in ruminants: From microbe to the farm scale. Animal 4:351-365.

Mejía, J. 2002. Consumo voluntario de forraje por rumiantes en pastoreo. Acta Universitaria 12(3):56-63.

Montenegro, J; Barrantes, E; DiLorenzo, N. 2018. Determinación de la emisión de metano entérico de novillos Brahman en pastoreo en el ecosistema de bosque tropical seco de Costa Rica. Trop. J. Environ. Sci. 52(2):158-170.

Montenegro, J; Barrantes, E; DiLorenzo, N. 2016. Methane emissions by beef cattle consuming hay of varying quality in the dry forest ecosystem of Costa Rica. Livestock Science 193:45-50.

Montenegro, J; Barrantes, E. 2016. Implementación de la técnica del hexafluoruro de azufre para cuantificar metano entérico en bovinos en Costa Rica. Trop. J. Environ. Sci. 50(2):62-74.
Moorby, J; Fleming, H; Theobald, V; Fraser, M. 2015. Can live weight be used as a proxy for enteric methane emissions from pasture-fed sheep? Scientific Reports 5:17915.

Moss, A; Jouany, J; Newbold, J. 2000. Methane production by ruminants: its contribution to global warming. INRA EDP Sciences, Ann Zootech 49:231-253.

Muñoz, C; Hube, S; Morales, J; Yan, T; Ungerfeld, E. 2015. Effects of concentrate supplementation on enteric methane emissions and milk production of grazing dairy cows. Livestock Science 175:37-46.

Steinfeld, H; Gerber, P; Wassenaar, T; Castel, V; Rosales, M; de Haan, C. 2006. Livestock's Long Shadow, Food and Agriculture Organization of the United Nations. 390 p.

Vlaming, J; Clark, H; Lopez-Villalobos, N. 2005. The effect of SF6 release rate, animal species and feeding conditions on estimates of methane emissions from ruminants. Proc. N.Z. Soc. Anim. Prod. 65:4-8.

Yan, T; Mayne, C; Gordon, F; Porter, M; Agnew, R; Patterson, D; Ferris, C; Kilpatrick, D. 2010. Mitigation of enteric methane emissions through improving efficiency of energy utilization and productivity in lactating dairy cows. J. Dairy Sci. 93:2630-2638. 